\title{
Respiratory Sinus Arrhythmia During Stress Predicts Resting Respiratory Sinus Arrhythmia 3 Years Later in a Pediatric Sample
}

\author{
Kristen Salomon \\ University of South Florida
}

\begin{abstract}
The author examined whether respiratory sinus arrhythmia (RSA) responses to stress predicted resting RSA approximately 3 years later in children and adolescents. A total of 149 children and adolescents (49\% girls and women, 44\% African Americans) participated in 2 laboratory protocols approximately 3 years apart. RSA reactivity during tasks was consistent within participants across tasks during each session. Resting RSA at Visit 1 explained $17 \%$ of the variance in resting RSA at Visit 2 when body mass index, duration between visits, race, gender, and age were controlled for. Visit 1 RSA reactivity explained an additional $5 \%$ of the variance in resting RSA at Visit 2 . The positive relationship between Visit 1 reactivity and Visit 2 resting levels suggests that larger decreases in RSA during stress predicted lower resting RSA. Conversely, increases in RSA during stress were associated with higher resting RSA an average of 3 years later.
\end{abstract}

Keywords: adolescents, cardiovascular reactivity, children, respiratory sinus arrhythmia, longitudinal design

The relationship of heart period variability (HPV) to cardiovascular disease (CVD) is supported by evidence demonstrating that low HPV is a prognostic indicator of mortality after a coronary event (for reviews, see Kristal-Boneh, Raifel, Froom, \& Ribak, 1995; van Ravenswaaij-Arts, Kollee, Hopman, Stoelinga, \& van Geijn, 1993) and of increased incidence of hypertension, coronary heart disease, and mortality (Dekker et al., 2000; Liao et al., 1997; Singh et al., 1998; Tsuji et al., 1996), as well as a correlate of risk factors for CVD (Friedman \& Thayer, 1998a, 1998b; Sloan et al., 1994). Most of this evidence is from researchers examining total HPV across both short-term and 24-hr recordings rather than the specific low- and high-frequency components. The high-frequency component of HPV assesses respiratory sinus arrhythmia (RSA) and is often used as an index of vagal control of the heart. Researchers have found evidence for RSA as a risk factor for CVD. Using data from the Atherosclerotic Risk in Communities study, Dekker et al. (2000) reported that participants with low RSA had increased relative risk of CVD mortality, both before and after adjustment for cardiovascular risk factors. The increased rate of CVD mortality was true for low-RSA participants with and without current clinical signs of CVD. Tsuji et al. (1996) reported that low RSA was related to increased risk of higher rates of coronary

This research was supported by National Institutes of Health Grant R01 HL25767-21 awarded to Karen A. Matthews, Professor of Psychiatry, Epidemiology, and Psychology, University of Pittsburgh, PA. I give special thanks to Dr. Matthews for providing the data and many helpful comments. I also thank Michael T. Allen and the rest of the dedicated staff members of the Child Health Study, which was conducted at the University of Pittsburgh School of Medicine.

Correspondence concerning this article should be addressed to Kristen Salomon, Department of Psychology, University of South Florida, 4202 East Fowler Avenue, PCD 4118G, Tampa, FL 33620. E-mail: ksalomon@chuma1.cas.usf.edu insufficiency, myocardial infarction, and death from both coronary heart disease and congestive heart failure after adjustment for clinical risk factors in these same samples. Therefore, low levels of the vagal component of HPV may be a prognostic risk factor for the development of CVD.

Vagal control serves to maintain homeostasis and promote growth and restoration. Porges and colleagues (Porges, 1995, 2001; Porges, Doussard-Roosevelt, Portales, \& Greenspan, 1996; Porges, Doussard-Roosevelt, Portales, \& Suess, 1994) proposed that vagal responses to stress function as a "brake" to quickly regulate responses to environmental demands. Further, high resting vagal control is an indicator of the behavioral and autonomic flexibility needed to react and self-regulate in response to environmental demands. In support of his model, Porges $(1991,1992)$ has shown that individuals with higher resting vagal control exhibit greater vagal withdrawal during sustained attention and stress; that infants with higher resting vagal control are more attentive to novel stimuli, are less distractible, and show faster habituation; and that adults with higher resting vagal control exhibit greater vagal withdrawal and better performance in a reaction time task. Thus, a corollary of Porges's (1991, 1992) model is that resting vagal control should predict vagal responses to stress. In turn, those with greater vagal responses to stress should exhibit greater resting vagal control. Resting vagal control as a predictor of later vagal responses to stress has been demonstrated (Porges, 1991, 1992, 1995, 2001; Thayer, Friedman, Borkovec, Johnson, \& Molina, 2000). To my knowledge, no other researchers to date have examined whether vagal responses to stress predict future resting vagal control.

\section{Individual Differences in RSA Reactivity}

Reactivity should predict later resting cardiovascular function under certain conditions (cf. Manuck, Kasprowicz, \& Muldoon, 
1990). First, reactivity should exhibit individual response stereotypy, that is, consistent individual differences in response to varied situations. Although differences across tasks in RSA reactivity have been found, individual differences among adults performing the same tasks also have been demonstrated (Berntson et al., 1994; Berntson, Cacioppo, \& Fieldstone, 1996; Berntson, Cacioppo, \& Quigley, 1991, 1993; Cacioppo, Uchino, \& Berntson, 1994). Specifically, during active coping tasks requiring cognitive efforts such as mental arithmetic, reaction time, and speech, participants' RSA on average decreased. However, a subset of individuals exhibited increases in RSA during these tasks (Berntson et al., 1991, 1993, 1994, 1996; Cacioppo et al., 1994). My colleagues and I have also found evidence for individual differences in RSA reactivity among children and adolescents (Salomon, Matthews, \& Allen, 2000). Although care must be taken when interpreting between-participants differences in RSA because of interindividual differences in mean heart rate (Grossman \& Kollai, 1993), the evidence does suggest that individual differences in vagal reactivity may be evident throughout the life span.

Second, individual differences in reactivity should be moderately stable across time, reflecting chronic response tendencies. This is not to say that reactivity is itself a trait. Instead, reactivity fits within a biopsychosocial model, suggesting that the interaction of social, personality, and biological factors within a person leads to stable reactivity tendencies. Third, stable individual differences in reactivity should be predictive of later resting levels (Manuck et al., 1990). A number of studies provide evidence for the stability of individual differences in vagal reactivity. Participants have exhibited moderate to high consistency of RSA reactivity within the same testing session and across different tasks (Berntson et al., 1994, 1996; Salomon et al., 2000; Sloan, Shapiro, Bagiella, Fishkin, et al., 1995). In a previous study (Salomon et al., 2000), my colleagues and I, using data that included the subset forming the present sample, reported correlations of approximately .78 between RSA reactivity across three different tasks given during the same testing session. Evidence for longitudinal stability across testing sessions has been found as well. Sloan, Shapiro, Bagiella, Gorman, and Bigger (1995) examined the temporal stability of RSA reactivity during two tasks repeated across three sessions in adults. They reported correlations of .27 for the individual tasks and .38 for combined-task RSA levels across testing sessions. A review of the literature revealed no studies to date in which researchers have examined longitudinal stability of RSA reactivity in children.

\section{Vagal Control in Children}

Researchers measuring RSA in children should take into account the known early developmental changes in vagal control of the heart. In general, children exhibit a fivefold increase in RSA during infancy and childhood and then exhibit a decline with age, reaching minimal levels between the ages of 70 and 79 (Korkushko, Shatilo, Plachinda, \& Shatilo, 1991; Umetani, Singer, McCraty, \& Atkinson, 1998). However, it is unclear at what point RSA reaches a maximum level. Some researchers have suggested that RSA peaks sometime between the ages of 4 and 6 (Finley \& Nugent, 1995; Yeragani, Pohl, Berger, Balon, \& Srinivasan, 1994), whereas others have found a peak between ages 10 and 14 (Korukushko et al., 1991; Massin \& von Bernuth, 1997). Although
RSA increases across childhood, significant stability of rank ordering has been demonstrated, with longitudinal correlations ranging from .36 to .55 (Bar-Haim, Marshall, \& Fox, 2000; Marshall \& Stevenson-Hinde, 1998; Porges et al., 1994). Although childhood and adolescence involve significant changes in vagal control of the heart, RSA tends to maintain in a stable trajectory across childhood and can be predictive of vagal control later in life.

The present data involve a follow-up of the sample of children and adolescents reported in Allen and Matthews (1997) and Salomon et al. (2000). Among the larger sample obtained at the initial visit, participants exhibited individual response stereotypy for RSA reactivity (Salomon et al, 2000). In the present follow-up sample, RSA reactivity was examined in respect to individual response stereotypy, stability, and prediction of later resting vagal control. I expected that participants would exhibit individual response stereotypy across tasks at Visit 2. In addition, I hypothesized that participants would exhibit stability of RSA across visits. Thus, I predicted that resting RSA at Visit 1 would be related to resting RSA at Visit 2, and RSA reactivity at Visit 1 would be related to RSA reactivity at Visit 2 . Finally, I hypothesized that RSA reactivity at Visit 1 would predict resting RSA at Visit 2, regardless of resting RSA at Visit 1.

\section{Method}

\section{Participants}

A total of 203 participants (124 children and 79 adolescents) were recruited from school districts in the metropolitan Pittsburgh, Pennsylvania, area for the initial phase (Visit 1) of the longitudinal study. Two participants who completed the laboratory protocol were excluded from the analyses, 1 because of a fever and 1 because of protocol problems. Participants in the child cohort were between 8 and 10 years old at Visit 1 and were prepubertal. Participants in the adolescent cohort were required to be between the ages of 15 and 17 at Visit 1 , and signs of late- to postpuberty were required for their involvement. Pubertal status was assessed with the interview version of the Petersen Pubertal Development Scale (Petersen, Crockett, Richards, \& Boxer, 1988). Because the goal was to recruit African American and European American participants of lower middle-class to middle-class families, participation was restricted to individuals whose parents did not have professional degrees.

Eligibility requirements were as follows: no history of CVD, mental illness, or any condition (e.g., high blood pressure, asthma, use of oral contraception) that requires medication that might affect the cardiovascular system; no drug or alcohol abuse and no professional psychiatric counseling in the past year; less than $80 \%$ above their ideal weight according to Metropolitan height and weight tables; and no smoking within $12 \mathrm{hr}$ prior to the session. The $80 \%$ below-ideal-weight criterion was imposed because of concerns about obtaining impedance cardiography signals of adequate fidelity with heavier children. Less than $2 \%$ of potential participants were excluded during initial screening because of weight.

Of the original 201 participants, 149 returned for the follow-up phase (Visit 2) of the study between 1.4 and 6.2 years later. Of the original group of 123 children, 100 returned, ranging in age at Visit 2 from 9 to 14 years Of the original 78 adolescents, 49 returned, ranging in age from 17 to 21 years. Fifty-two participants did not return (24 were not invited back because they had not participated in the voluntary echocardiogram at Visit 1, 14 could not be located, 13 declined follow-up participation, and 1 was deceased). The final distribution for Visit 2 contained 24 African American female children, 25 European American female children, 22 African American male children, 29 European American male children, 9 African American female adolescents, 15 European American female adolescents, 
10 African American male adolescents, and 15 European American male adolescents.

\section{Physiological Recording Apparatus}

The electrocardiogram (ECG) was used for the measurement of heart periods to compute RSA with the mean successive difference (MSD) statistic. The ECG signal was transduced using two active and one ground Cleartrace LT disposable silver-silver chloride electrodes (Conmed Andover Medical, Haverhill, MA) with a modified Lead II configuration. The ECG signal was filtered and amplified by a Coulbourn S75-11 amplifier/ coupler (Coulbourn Instruments, Allentown, PA). Impedance cardiography and blood pressure were also collected. However, those data are not presented here.

Processing of the ECG and impedance signals was accomplished with the Cardiac Output Program (COP; Microtronics, Chapel Hill, NC), an online computerized video graphics system for ECG and impedance cardiography analysis. Basal impedance, the first derivative of the pulsatile impedance signal (dZ/dt) and the ECG, was sampled at $500 \mathrm{~Hz}$ per channel with a personal computer-based microcomputer hosting an analog-todigital converter board. Details of the calculations of the various physiological measures from impedance cardiography can be found in Sherwood et al. (1990).

\section{Experimental Tasks}

At Visit 1, participants completed four reactivity tasks: reaction time, mirror tracing, Social Competence Interview (SCI; Ewart \& Kolodner, 1991), and forehead cold pressor. At Visit 2, participants engaged in three of these tasks; the interview was not repeated at Visit 2. Data from the forehead cold pressor tasks were not used in the present analyses, as the data were collected in such a way to make interpretation of HPV suspect. Therefore, the forehead cold pressor task is not discussed further in this article.

Reaction time. A computerized choice reaction time task required the participant to respond as quickly as possible to a $1000-\mathrm{Hz}$ tone presented via headphones by pressing a joystick button but to refrain from responding to a 2000-Hz tone. During the 3-min task, a PC presented tones at irregular intervals. Participants received a monetary reward, with the amount depending on their performance. On average, participants received $\$ 5$ for the task.

Mirror tracing. Participants were required to trace around a copper star with a metal stylus while only being allowed to see the mirror image of the star. The tracing apparatus (Catalog No. 31213, Stoelting, Chicago, IL) was interfaced to a PC, and customized software kept track of the task time and whether the stylus was on the star. Going off the star produced a loud beep through the headphones. The task time was 3 min.

SCI. Participants were administered the SCI by a trained interviewer. Participants taking the SCI are encouraged to describe and discuss an interpersonal source of distress for at least $10 \mathrm{~min}$ but for no longer than 15 min. Ewart and Kolodner (1991) have shown that this task elicits relatively large increases in blood pressure and heart rate. Physiological responses were collected during the first $10 \mathrm{~min}$ of the task.

\section{Experimental Protocol}

The protocol was explained to the parents in detail during the initial recruitment contact. Adolescents and their parents were required to sign a consent form prior to their participation in the protocol (the younger children signed an assent form, and their parents signed a separate consent form). The Institutional Review Board of the University of Pittsburgh Medical Center, Pennsylvania, approved all consent and assent forms.

Participants arrived at the laboratory at about 8:30 a.m. after an overnight fast and fluid restriction. Height, weight, and skin folds were mea- sured after the participant changed into a hospital gown. A venous blood draw was then performed for a variety of biochemical assays (not reported here). After the blood draw, the participants were given a light breakfast, then electrodes and transducers were applied for ECG, impedance cardiography, and blood pressure measurement. Participants were then given instructions to relax for an initial 10-min rest period.

The reactivity tasks were given in a counterbalanced order with 8-min intertask rest periods. A final 10-min rest period followed the last task. Sensors were removed following the last rest period, and participants were then given a number of psychosocial questionnaires, the results of which are not reported here. Participants were paid $\$ 75$ for completing the protocol, along with money earned on the reaction time task.

\section{Data Reduction}

ECG data were collected on a minute-by-minute basis during the last 3 min of the initial and final rest periods, during the last minute of the intertask rest periods, during the entire $3 \mathrm{~min}$ of reaction time and mirror tracing tasks, and during the first $10 \mathrm{~min}$ of the SCI. COP allowed for collection of $55 \mathrm{~s}$ of each minute, with $5 \mathrm{~s}$ of the minute needed for writing the file to disk. Customized software allowed me to extract continuous heart period data from files created by COP. The data were screened and edited for artifactual values, and the MSD was computed for each data series. MSD was obtained by calculating the absolute difference between successive heart periods and then averaging those differences over a particular time period.

MSD was used on the basis of evidence that it estimates beat-to-beat variability in the respiratory frequency range and correlates with vagally mediated heart rate variability (Fox, 1983; Hayano et al., 1991; The Task Force of the European Society of Cardiology and the North American Society of Pacing and Electrophysiology, 1996; Yeh, Forsythe, \& Hon, 1973). As a differencing technique, low-frequency sources of variability in the heart period series, such as linear trends and very slow oscillations, are filtered out. MSD tracks pharmacologically manipulated cardiac vagal control. Hayano et al. (1991) defined vagal cardiac control as the change in mean interbeat interval from sympathetic to full autonomic blockade during paced breathing at $0.25 \mathrm{~Hz}$. MSD correlated at .92 with this index of vagal cardiac control, similar in magnitude to the .91 correlation obtained between high-frequency spectral power and vagal cardiac control (Hayano et al., 1991)

\section{Data Analyses}

The initial rest period was selected as the baseline period because the majority of cardiovascular measures were lowest during this period. I computed reactivity scores by subtracting the baseline value from the task value. To create an aggregate measure of reactivity across the three tasks at Visit 1, I performed $z$ transformations of the reactivity scores and then averaged those scores.

For multiple regression analyses, I first examined the effects of age, race, and gender and their interaction on Visit 2 resting RSA using the procedures outlined in Jaccard, Turrisi, and Wan (1990). To examine the effects of Visit 1 RSA reactivity on Visit 2 resting RSA levels, I performed a hierarchical analysis, regressing Visit 1 resting RSA and Visit 1 averaged standardized task reactivity on Visit 2 resting RSA while controlling for body mass index (BMI); duration between visits; age; race; and gender. In regression equations involving interaction terms, continuous variables were centered on their means.

\section{Results}

I examined the distributions for resting RSA, RSA reactivity for each task, and averaged RSA reactivity. I identified 2 participants whose values for reaction time, mirror tracing, and averaged RSA 
reactivity were outliers ( $>3$ standard deviations from the mean) and removed their data from the analyses. This reduced the skew for these variables such that the skew for all continuous variables was less than 1 in absolute value.

I performed independent sample $t$ tests comparing the remaining 147 who participated and the 28 eligible nonparticipants on Visit 1 resting RSA, on RSA reactivity averaged across tasks, and for each task separately. Participants did not differ significantly from nonparticipants in Visit 1 resting RSA or mirror tracing and interview RSA reactivity (all $p s>.14$ ). There was a marginally significant trend, suggesting that participants exhibited greater decreases in RSA to the reaction time task than did nonparticipants at Visit $1, t(173)=-1.88, p=.06$. Comparisons of Visit 2 participants with those who were not invited to participate $(n=22$; see eligibility criteria) revealed no significant differences in resting RSA or RSA reactivity at Visit 1 (all $p \mathrm{~s}>.09$ ).

The relationship between resting RSA and RSA reactivity was examined within visits. At Visit 1, resting RSA was correlated with change in RSA $(r \mathrm{~s}=-.60,-.69$, and -.63 , all $p \mathrm{~s}<.01$, during the reaction time task, the mirror tracing task, and the interview, respectively). At Visit 2, resting RSA was correlated with change in RSA $(r \mathrm{~s}=-.36$ and $-.45, p \mathrm{~s}<.01$, during the reaction time and mirror tracing tasks, respectively). These correlations show that greater resting RSA was predictive of larger decreases in RSA during the tasks, particularly at Visit 1.

I calculated means and standard deviations for RSA at baseline and tasks for groups by age, gender, and ethnicity. These are presented in Table 1 for descriptive purposes. On average, the SCI produced the largest changes in RSA at Visit 1, whereas the reaction time task produced the smallest changes at both visits. Similar to prior analyses (Salomon et al., 2000), the percentage of participants who exhibited increases in RSA was examined for each task. These percentages for the reaction time task, mirror tracing task, and interview were $22.4 \%, 15.6 \%$, and $11.1 \%$, re- spectively. Pearson product-moment correlation coefficients between pairs of tasks were computed to examine the consistency of RSA reactivity at each visit. At Visit 1 , the correlation between reaction time and mirror tracing was $.75(p<.01)$; between reaction time and interview, $.76(p<.01)$; and between mirror tracing and interview, .74 $(p<.01)$. At Visit 2, the correlation between reaction time and mirror tracing was .68, $p<.01$.

I performed correlations between Visit 1 and Visit 2 RSA levels. Resting RSA was correlated at $.48(p<.01)$, between visits. Table 2 presents Pearson product-moment correlation coefficients between RSA reactivity at Visit 1 and Visit 2. Within-task correlations between RSA reactivity across time were small. Reaction time reactivity at Visit 1 correlated significantly with reaction time and mirror tracing reactivity at Visit 2 . All other individual task correlations were not significant. The correlation between the three-task aggregate reactivity scores was also nonsignificant $(r=$ $.10, p=.23)$. I created an aggregate reactivity score including only reaction time and mirror tracing reactivity at Visit 1 , as only these two tasks were repeated at Visit 2. The correlation between the two-task aggregate reactivity scores was $.17(p=.04)$.

Age, gender, ethnicity effects, and their interactions were regressed on Visit 2 resting RSA. BMI and duration between visits were included as predictors on Step 1, but these variables were not included in any interactions because none were expected or predicted. Gender was a significant predictor of Visit 2 resting RSA, with boys and men exhibiting greater RSA than did girls and women. The variance accounted for by BMI, duration, age, gender, and ethnicity was $11.1 \%$, and the multiple correlation squared was significant, $F(5,139)=3.48, p=.005$. Step 2 included the three bivariate interactions among age, gender, and ethnicity. No interaction was significant, and this step did not account for a significant amount of additional variance, $\Delta F(3,136)=0.70, p=.55$. Step 3 added the three-way interaction among age, race, and ethnicity and was also not significant, $\Delta F(1,133)=0.17, p=.68$.

Table 1

Mean Baseline Levels and Reactivity Scores of RSA

\begin{tabular}{|c|c|c|c|c|c|c|c|c|c|c|c|c|c|c|c|c|}
\hline \multirow[b]{3}{*}{ Visit } & \multicolumn{8}{|c|}{ Girls and adolescent women } & \multicolumn{8}{|c|}{ Boys and adolescent men } \\
\hline & \multicolumn{4}{|c|}{ African American } & \multicolumn{4}{|c|}{ European American } & \multicolumn{4}{|c|}{ African American } & \multicolumn{4}{|c|}{ European American } \\
\hline & B & RT & MR & SCI & B & RT & MR & SCI & B & RT & MR & SCI & B & RT & MR & SCI \\
\hline \multicolumn{17}{|c|}{ Visit 1} \\
\hline \multicolumn{17}{|l|}{ Children } \\
\hline$M$ & 65.3 & -13.5 & -22.1 & -22.5 & 45.9 & -6.8 & -12.2 & -17.6 & 65.1 & -17.6 & -20.9 & -27.0 & 55.8 & -11.5 & -13.6 & -17.0 \\
\hline$S D$ & 39.3 & 13.5 & 22.7 & 16.7 & 24.1 & 15.1 & 15.9 & 16.0 & 41.2 & 23.3 & 25.3 & 29.7 & 30.6 & 25.3 & 24.7 & 30.4 \\
\hline \multicolumn{17}{|c|}{ Adolescents } \\
\hline$M$ & 68.7 & -27.2 & -28.8 & -22.0 & 63.9 & -19.1 & -23.5 & -34.2 & 74.7 & -24.7 & -22.6 & -19.3 & 69.1 & -8.8 & -8.4 & -13.8 \\
\hline$S D$ & 42.3 & 27.1 & 19.9 & 25.1 & 38.6 & 27.6 & 28.0 & 30.1 & 24.0 & 23.4 & 14.3 & 20.7 & 38.8 & 20.9 & 22.3 & 35.7 \\
\hline \multicolumn{17}{|c|}{ Visit 2} \\
\hline \multicolumn{17}{|l|}{ Children } \\
\hline$M$ & 50.7 & -7.6 & -10.1 & & 35.3 & 0.3 & -2.5 & & 60.1 & -2.3 & -5.5 & & 55.2 & -1.2 & -6.8 & \\
\hline$S D$ & 29.3 & 20.2 & 19.1 & & 16.1 & 14.1 & 12.5 & & 32.1 & 22.2 & 16.4 & & 21.6 & 15.3 & 19.0 & \\
\hline \multicolumn{17}{|l|}{ Adolescents } \\
\hline$M$ & 55.4 & -4.9 & -7.8 & & 48.6 & -2.0 & -6.6 & & 60.9 & -19.9 & -21.2 & & 59.7 & -9.2 & -9.7 & \\
\hline$S D$ & 30.7 & 21.2 & 17.7 & & 28.1 & 11.8 & 24.2 & & 24.6 & 19.6 & 17.2 & & 28.9 & 17.8 & 10.2 & \\
\hline
\end{tabular}

Note. $\mathrm{RSA}=$ respiratory sinus arrhythmia; $\mathrm{B}=$ baseline; $\mathrm{RT}=$ reaction time; $\mathrm{MR}=$ mirror tracing; $\mathrm{SCI}=$ Social Competence Interview. 
Table 2

Correlations Between RSA Reactivity Across Time

\begin{tabular}{llllll}
\hline & \multicolumn{4}{c}{ Visit 2 } \\
\cline { 2 - 3 } \cline { 5 - 6 } \multicolumn{1}{c}{ Visit 1} & \multicolumn{2}{c}{ Reaction time } & & \multicolumn{2}{c}{ Mirror tracing } \\
\cline { 2 - 3 } \cline { 5 - 6 } & $r$ & $n$ & & $r$ & $n$ \\
\hline Reaction time & $.21^{* *}$ & 145 & & $.18^{*}$ & 145 \\
Mirror tracing & .06 & 145 & & .13 & 145 \\
Interview & -.03 & 142 & & .03 & 142 \\
\hline
\end{tabular}

Note. $\mathrm{RSA}=$ respiratory sinus arrhythmia.

$* p<.05$. ** $p<.01$.

The results of the regression analyses examining the effects of Visit 1 RSA on Visit 2 resting RSA are presented in Table 3. Step 1 of this analysis was identical to Step 1 of the previous regression analysis. Although BMI, duration, age, and ethnicity were not independent significant predictors in the first regression analysis, their effects were not zero, and thus I included them again. On Step 2, Visit 1 resting RSA accounted for an additional $17.1 \%$ of the variance in Visit 2 resting RSA, and the multiple correlation squared change was significant, $\Delta F(1,138)=32.94, p<.01$. On Step 3, mean standardized RSA reactivity accounted for an additional $4.7 \%$ of the variance in Visit 2 resting RSA, which was significant, $\Delta F(1,137)=10.67, p=.002 .{ }^{1}$ On Step 4 , the bivariate interactions among RSA reactivity and age, gender, and ethnicity were examined. ${ }^{2}$ This step did not account for a significant amount of additional variance. The interaction between gender and Visit 1 RSA reactivity approached significance, $t(138)=$ $-1.93, p=.056$, suggesting that the slope for boys and adolescent men was smaller than the slope for girls and adolescent women. Tests of the simple slopes revealed that the slopes for both female and male participants were significant, $t(63)=3.45, p=.001$, and $t(68)=2.20, p=.029$, respectively.

The predictive value of RSA was examined for each task individually. I performed three separate regression analyses that were parallel to the aggregate reactivity model, except that on Step 3 I entered reactivity for only one of the three tasks. Age, race, gender, BMI, duration between visits, and Visit 1 resting RSA were entered on Step 1. On Step 3 of their respective regression analyses, Visit 1 mirror tracing and RSA reactivity of the SCI accounted for a significant amount of variance in Visit 2 resting RSA. Visit 1 mirror tracing RSA accounted for $5.5 \%$ of variance in Visit 2 resting RSA, $\Delta F(1,137)=11.47, p=.001$, and RSA of the SCI accounted for $4.6 \%$ of variance, $\Delta F(1,134)=9.28, p=$ .003 . However, Visit 1 reaction time RSA levels did not significantly predict Visit 2 resting $\mathrm{RSA}, \Delta F(1,137)=2.44, p=.12$.

\section{Discussion}

In the present study, I examined whether RSA reactivity predicted resting RSA approximately 3 years later in children and adolescents. The results supported my predictions. First, initial resting RSA accounted for $17 \%$ of the variance in resting RSA from 1.5 to 5.0 years later. This finding is compelling considering the age of the sample and the known developmental changes in RSA. This finding also supports Porges and colleagues' (Porges, 1995, 2001; Porges et al., 1994, 1996) contention that vagal control predicts vagal control. Further, RSA reactivity predicted later resting RSA, when controlling for initial resting RSA, age, gender, ethnicity, BMI, and duration between visits. Reactivity accounted for approximately $5 \%$ of the variance in Visit 2 resting RSA, which was a smaller effect than in Visit 1 resting RSA but still meaningful in light of the sensitivity of this predictor (Prentice $\&$ Miller, 1992). In short, reactivity measured during three short tasks on a single visit predicted resting level approximately 3 years later.

The nature of the relationship between Visit 1 reactivity and Visit 2 resting level was such that a larger decrease in RSA during stress was related to lower resting RSA. Although children on average showed decreases in RSA during these tasks, approximately $11 \%$ to $22 \%$ of children exhibited RSA increases. Thus, children who tend to show RSA increases or smaller decreases during stress may have the benefit of higher levels of cardiac vagal control on entering young adulthood. Research shows that RSA decreases steadily after adolescence (Korukushko et al., 1991), suggesting that those with active vagal brakes may begin this decline from a higher starting point. Whether the predictive-value RSA reactivity is evident in adult samples remains to be tested.

The present findings also confirmed that RSA reactivity is relatively consistent in a child sample across a variety of tasks. Participants exhibited similar within-session RSA reactivity across tasks at Visit 1 (Salomon et al., 2000) and at Visit 2. The magnitudes of the within-session reactivity correlations are similar to correlations found with adults (Berntson et al., 1994; Sloan, Shapiro, Bagiella, Fishkin, et al., 1995; Sloan, Shapiro, Bagiella, Gorman, \& Bigger, 1995). However, the present across-sessions stability estimates were lower than those reported by Sloan and colleagues in adults measured at $0,1,4$, and 9 months after the initial study assessment (Sloan, Shapiro, Bagiella, Gorman, \& Bigger, 1995). Resting RSA stability estimates in the present study may have been lower because they were based on fewer assess-

\footnotetext{
${ }^{1}$ Because task levels are often highly correlated with resting levels, I examined the issue of multicollinearity in regard to including both Visit 1 resting and Visit 1 reactivity in the same regression equation. The correlation between these was - .72. As Tabachnick and Fidell (1989) suggested, multicollinearity problems occur with much higher correlations $(r \geq .90)$. The tolerances were both .490 for Visit 1 resting RSA and Visit 1 aggregate RSA reactivity when both were in the model. Again, although these were not as high as the other variables in the equation (with tolerances ranging from .570 for age to .963 for ethnicity), they were not so low as to suggest serious multicollinearity problems, as would a tolerance close to .010 .

${ }^{2}$ I also conducted these regression analyses separately for the children and adolescents because the overall sample consisted of twice as many children as adolescents. Results for the children were essentially the same as those for the overall analysis, with Visit 1 resting RSA and Visit 1 reactivity both explaining a significant amount of additional variance in Visit 2 resting RSA. For adolescents, Visit 1 resting RSA accounted for a significant amount of variance in Visit 2 resting RSA, but Visit 1 reactivity did not. This is likely due to a decrease in power, as there were half as many adolescents as children. The standardized regression coefficients for Visit 1 reactivity predicting Visit 2 resting for children $(\beta=.28)$ and adolescents $(\beta=.22)$ were similar in magnitude. Further, the lack of a significant interaction between age and Visit 1 reactivity in the analysis combining children and adolescents indicates that these slopes were not significantly different.
} 
Table 3

Regression Results for Visit 1 (V1) Resting RSA and RSA Reactivity Predicting Visit 2 (V2)

Resting RSA, While Controlling for BMI, Duration Between Visits, Age, Gender, and Ethnicity

\begin{tabular}{|c|c|c|c|c|c|c|c|}
\hline \multirow[b]{2}{*}{ Variable } & \multicolumn{7}{|c|}{ V2 Resting RSA } \\
\hline & $b$ & $S E b$ & $\beta$ & $\Delta R^{2}$ & $d f$ & $F$ & $p$ \\
\hline Step 1 & & & & .11 & 5,139 & 3.48 & .005 \\
\hline BMI & -1.01 & 0.60 & -.16 & & & & \\
\hline Duration between V1 and V2 & -0.09 & 2.93 & .003 & & & & \\
\hline Age at V2 (centered) & 1.29 & 0.77 & .17 & & & & \\
\hline Gender (female $=0$ ) & 13.50 & 4.34 & $.26^{* *}$ & & & & \\
\hline Ethnicity (African American = 0) & -6.70 & 4.25 & -.13 & & & & \\
\hline Step 2 & & & & .17 & 1,138 & 32.94 & $<.01$ \\
\hline V1 Resting RSA & 0.34 & 0.06 & $.43 * *$ & & & & \\
\hline Step 3 & & & & .05 & 1,137 & 9.67 & .002 \\
\hline V1 RSAR & 9.73 & 3.13 & $.31 * *$ & & & & \\
\hline Step 4 & & & & .02 & 3,134 & 1.34 & .260 \\
\hline Age $\times$ RSAR & -0.72 & 0.75 & -.08 & & & & \\
\hline Gender $\times$ RSAR & -9.27 & 4.80 & $-.23 \dagger$ & & & & \\
\hline Ethnicity $\times$ RSAR & -0.45 & 4.86 & -.01 & & & & \\
\hline
\end{tabular}

Note. $\quad$ RSA $=$ respiratory sinus arrhythmia; $\mathrm{BMI}=$ body mass index; $\mathrm{b}=$ unstandardized regression coefficient; RSAR $=$ RSA reactivity standardized for each task and averaged across tasks

$\dagger p<.06 . \quad * * p<.01$.

ments at a longer time interval. RSA may also be more sensitive to proximal environmental factors. As environments change over time, so might individual response tendencies of vagal reactivity. Despite the low temporal stability of RSA reactivity, it predicted later resting RSA. To my knowledge, this study is the first to demonstrate the temporal stability of RSA reactivity in children and adolescents.

Significant gender differences in RSA at Visit 2 were observed. Boys and adolescent men exhibited higher resting RSA than did girls and adolescent women. These gender differences in resting RSA were not observed at Visit 1 in the larger sample of 201 youth (Allen \& Matthews, 1997). Research on adult gender differences in vagal control has been inconsistent. Some researchers have reported that women exhibit greater RSA than do men (Huikuri et al., 1996; Mäkimattila et al., 2000; Rossy \& Thayer, 1998; Sinnreich, Kark, Friedlander, Sapoznikov, \& Luria, 1998), whereas others have reported no gender differences (Bigger et al., 1995; Liao et al., 1995; Sevre et al., 2001; Stein, Kleiger, \& Rottman, 1997). In one study including children, boys and men exhibited greater RSA than did girls and women in the 10 to 29 age group, but at ages 30 and above, the RSAs of men and women did not differ. All of the studies reporting greater RSAs for adult women included participants in their early 20s. The present results regarding gender differences are consistent with the hypothesis that into their 20s, girls and women exhibit lower RSA than do boys and men, although these differences may disappear or reverse at older ages.

In the present study, greater resting RSA predicted greater decreases during stress and greater resting RSA approximately 3 years later. In contrast to Porges's $(1995,2001)$ model, greater vagal decreases during stress were not associated with greater adaptive functioning. Conversely, smaller decreases during stress - and in some cases, increases - predicted higher levels of vagal control in the future and greater self-regulation in the form of less family conflict (Salomon et al., 2000). Much of Porges's
(1995, 2001) work, as well as the work of others (Calkins, 1997; El-Sheikh, 2001; El-Sheikh, Harger, \& Whitson, 2001), has demonstrated that larger decreases in vagal control during stress are related to adaptive functioning.

There are two possible explanations for the inconsistency between earlier findings and those of this study. First, researchers in the aforementioned studies examined adaptive behavioral outcomes, whereas in the present study, I addressed adaptive cardiovascular outcomes. Adaptive behavior is not necessarily synonymous with adaptive cardiovascular response. Second, differences among study tasks may explain the discrepancy among study findings. El-Sheikh et al. (2001) measured vagal reactivity in children while they listened to adults' arguments. This type of task requires vigilance - an active monitoring of the environment for meaningful and possibly dangerous information and a readiness for quick response. For vigilance tasks, decreases in vagal control allow for sustained attention and preparation for quick spontaneous action. On the other hand, increases in vagal control are related to soothing and calming behaviors, as Porges $(1995,2001)$ notes. For nonvigilance tasks, smaller vagal decreases or vagal increases may facilitate controlled behavioral responses by facilitating soothing and calming. In the present sample, RSA reactivity during a vigilance task (i.e., reaction time) was not a significant independent predictor of later resting vagal control. Sahar, Shalev, \& Porges's (2001) work supports this idea. Compared with patients with posttraumatic stress disorder, participants with a past trauma but no posttraumatic stress disorder symptomatology exhibited increases in vagal control during a mental arithmetic task (Sahar et al., 2001), suggesting that vagal increases were indicative of more adaptive responses to stress. Alternatively, reaction time RSA reactivity was smaller than mirror tracing and interview reactivity in the present sample (see Table 1), and smaller changes in RSA may not have the same predictive relationship to later resting RSA. 
The present findings also suggest that RSA reactivity may alter the set point for resting RSA. Dworkin $(1993,2000)$ proposed that the autonomic nervous system exhibits adaptive changes through conditioning to anticipate future homeostatic disturbances. Encountering stimuli that perturb a resting state activates mechanisms to regulate the autonomic nervous system and maintain homeostasis. These mechanisms, such as the baroreflex response, create changes in the system that eventually return it to the original resting state. Through repeated exposure to perturbing stimuli and concurrent activation of higher order brain system responses to stimulus features, the autonomic system may anticipate future disturbances and adjust the resting state appropriately (Dworkin, 1993, 2000). That is, repeated changes in vagal activity during stressful situations could lead to long-term changes in resting vagal control, reflecting anticipation of future encounters with these situations. Individuals with a tendency toward vagal increases during stress should exhibit increases in resting vagal control, whereas individuals with large decreases during stress should exhibit decreases in resting vagal control. Through this process of conditioning, vagal control is regulated to a level closer to stress response tendencies.

The present results should be interpreted in light of a number of considerations. Respiration was not controlled for because breathing was neither measured nor paced. Researchers have found that within-participant changes in respiration influence changes in RSA that are independent of changes in vagal control (Grossman, Karemaker, \& Wieling, 1991; Houtveen, Rietveld, \& De Geus, 2002; Taylor, Myers, Halliwill, Seidel, \& Eckberg, 2001). Other researchers have mitigated this concern, showing that correcting for changes in respiratory rate from rest to tasks may not yield an optimal estimate of changes in vagal control and that RSA reactivity corrected for respiration rate parallels uncorrected RSA reactivity under nonpaced breathing conditions (Houtveen et al., 2002). However, RSA is also influenced by respiratory depth and central respiratory drive, which have also been shown to independently influence RSA (Grossman et al., 1991; Grossman \& Kollai, 1993; Houtveen et al., 2002; Taylor et al., 2001). Therefore, the possibility remains that the present findings are influenced by changes in respiration, not by changes in vagal control of the heart.

Lack of respiratory control presents another consideration. Validation of MSD with pharmacological blockade and spectral estimates has occurred primarily under conditions of paced breathing. In one exception, correlations between MSD and spectral estimates of RSA under conditions of nonpaced breathing averaged .75 (Friedman, Allen, Christie, \& Santucci, 2002), suggesting a high degree of overlap in the two estimates. Further, similar patterns of results have been found across measures within the same study under conditions of nonpaced breathing (Thayer, Friedman, \& Borkovec, 1996).

A related consideration is the use of MSD as a measure of RSA. Heart period affects the number of samples included in MSD, which may bias the statistic. However, recent evidence supports the idea that estimates of MSD that are corrected for heart period sampling bias are highly correlated with uncorrected MSD $(r=$ .98; Friedman et al., 2002). Another criticism of MSD is that as a successive beat measure, MSD captures variability around the frequency of heart rate and misses variability at the vagally mediated frequencies below $1.00 \mathrm{~Hz}$. However, MSD correlates highly with vagal cardiac control at an RSA frequency of $0.25 \mathrm{~Hz}$ under conditions of paced breathing (Fox, 1983; Hayano et al., 1991; Thayer et al., 1996, 2000; Yeh et al., 1973). In light of these considerations, these latter findings suggest that MSD seems to adequately reflect the respiratory-mediated effects of vagal activity on the heart. Further, MSD as an index of respiratory-mediated vagal influences on the end organ are precisely the effects that should be relevant to the role of RSA as a marker for the development of CVD.

More generally, regardless of the statistic used, care must be taken when interpreting interindividual differences in RSA, such as the gender differences reported here. As Grossman and Kollai (1993) have demonstrated, individual estimates of RSA based on HPV are influenced by both vagal tone and mean heart rate. RSA can only estimate vagal modulation of heart rate and not absolute vagal control. As such, comparisons among individuals on RSA may not truly reflect absolute differences among individuals in vagal control.

From a CVD perspective, individual differences in vagally mediated reactivity may predict differences in future resting vagal control and risk for CVD, similar to the predictive relationship of blood pressure reactivity on later resting blood pressure (for evidence supporting this relationship, see Carroll et al., 2001; Matthews, Salomon, Kenyon, \& Allen, 2002; Murphy, Alpert, Walker, \& Willey, 1991; Newman, McGarvey, \& Steele, 1999; Parker et al., 1987; Treiber et al., 2001). Within both adaptive selfregulation and CVD frameworks, in the present research I examined RSA responses to three tasks in children and adolescents as predictors of resting RSA control an average of 3 years later. I hypothesized that RSA increases during stress predict higher resting RSA, just as exaggerated blood pressure increases are related to higher resting blood pressure. Because higher resting vagal control may be related to better cardiovascular health, I suggested an adaptive reactivity hypothesis, in which vagal increases during stress are related to lower CVD risk.

Although results indicate that both resting RSA and RSA reactivity predict resting RSA over approximately 1 to 5 years in children and adolescents, the proposition that RSA reactivity is related to CVD risk is speculative. It is important to note, however, that RSA reactivity predicted higher resting RSA beyond what was predicted by resting RSA alone. In other words, reactivity did not merely protect from the deleterious effects of stress but seems to have afforded an advantage in an additional boost to resting vagal control. Dienstbier (1989), in his model of physiological toughness, proposed that exposure to intermittent stressors leads to decreased basal sympathetic nervous system arousal, in essence promoting health by rendering an individual more physiologically hardy. The present findings also suggest that exposure to stressors can lead to increased basal parasympathetic nervous system arousal, resulting from an adaptive parasympathetic stress response.

\section{References}

Allen, M. T., \& Matthews, K. A. (1997). Hemodynamic responses to laboratory stressors in children and adolescents: The influences of age, race, and gender. Psychophysiology, 34, 329-339.

Bar-Haim, Y., Marshall, P. J., \& Fox, N. A. (2000). Developmental changes in heart period and high-frequency heart period variability from 4 months to 4 years of age. Developmental Psychobiology, 37, 44-56. 
Berntson, G. G., Cacioppo, J. T., Binkley, P. F., Uchino, B. N., Quigley, K. S., \& Fieldstone, A. (1994). Autonomic cardiac control: III. Psychological stress and cardiac response in autonomic space as revealed by pharmacological blockades. Psychophysiology, 31, 599-608.

Berntson, G. G., Cacioppo, J. T., \& Fieldstone, A. (1996). Illusions, arithmetic, and the bidirectional modulation of vagal control of the heart. Biological Psychology, 44, 1-17.

Berntson, G. G., Cacioppo, J. T., \& Quigley, K. S. (1991). Autonomic determinism: The modes of autonomic control, the doctrine of autonomic space, and the laws of autonomic constraint. Psychological Review, 98, 459-487.

Berntson, G. G., Cacioppo, J. T., \& Quigley, K. S. (1993). Cardiac psychophysiology and autonomic space in humans: Empirical perspectives and conceptual implications. Psychological Bulletin, 114, 296322.

Bigger, J. T., Fleiss, J. L., Steinman, R. C., Rolnitzky, L. M., Schneider, W. J., \& Stein, P. K. (1995). RR variability in healthy, middle-aged persons compared with patients with chronic coronary heart disease or recent acute myocardial infarction. Circulation, 91, 1936-1943.

Cacioppo, J. T., Uchino, B. N., \& Berntson, G. G. (1994). Individual differences in the autonomic origins of heart rate reactivity: The psychometrics of respiratory sinus arrhythmia and preejection period. Psychophysiology, 31, 412-419.

Calkins, S. D. (1997). Cardiac vagal tone indices of temperamental reactivity and behavioral regulation in young children. Developmental Psychobiology, 31, 125-135.

Carroll, D., Smith, G. D., Shipley, M. J., Steptoe, A., Brunner, E. J., \& Marmot, M. G. (2001). Blood pressure reactions to acute psychological stress and future blood pressure status: A 10-year follow-up of men in the Whitehall II study. Psychosomatic Medicine, 63, 737-746.

Dekker, J. M., Crow, R. S., Folsom, A. R., Hannan, P. J., Liao, D., Swenne, C. A., et al. (2000). Low heart rate variability in a 2-minute rhythm strip predicts risk of coronary heart disease and mortality from several causes: The ARIC Study. Circulation, 102, 1239-1244.

Dienstbier, R. A. (1989). Arousal and physiological toughness: Implications for mental and physical health. Psychological Review, 96, 84-100.

Dworkin, B. R. (1993). Learning and physiological regulation. Chicago: University of Chicago Press.

Dworkin, B. R. (2000). Interoception. In J. T. Cacioppo, L. G. Tassinary, \& G. G. Berntson (Eds.), Handbook of psychophysiology (2nd ed., pp. 482-506). Cambridge, England: Cambridge University Press.

El-Sheikh, M. (2001). Parental drinking problems and children's adjustment: Vagal regulation and emotional reactivity as pathways and moderators of risk. Journal of Abnormal Psychology, 110, 499-515.

El-Sheikh, M., Harger, J., \& Whitson, S. M. (2001). Exposure to interparental conflict and children's adjustment and physical health: The moderating role of vagal tone. Child Development, 72, 1617-1636.

Ewart, C. K., \& Kolodner, K. B. (1991). Social competence interview for assessing physiological reactivity in adolescents. Psychosomatic Medicine, 53, 289-304.

Finley, J. P., \& Nugent, S. T. (1995). Heart rate variability in infants, children, and young adults. Journal of the Autonomic Nervous System, 51, 103-108.

Fox, N. A. (1983). Maturation of autonomic control in preterm infants. Developmental Psychobiology, 16, 495-504.

Friedman, B. H., Allen, M. T., Christie, I. C., \& Santucci, A. K. (2002). Validity concerns of common heart-rate variability indices: Addressing quantification issues in time- and frequency-domain measures of HRV. IEEE Engineering in Medicine and Biology, 21, 35-40.

Friedman, B. H., \& Thayer, J. F. (1998a). Anxiety and autonomic flexibility: A cardiovascular approach. Biological Psychology, 49, 303-323.

Friedman, B. H., \& Thayer, J. F. (1998b). Autonomic balance revisited: Panic anxiety and heart rate variability. Journal of Psychosomatic Research, 44, 33-51.
Grossman, P., Karemaker, J., \& Wieling, W. (1991). Prediction of tonic parasympathetic cardiac control using respiratory sinus arrhythmia: The need for respiratory control. Psychophysiology, 30, 201-216.

Grossman, P., \& Kollai, M. (1993). Respiratory sinus arrhythmia, cardiac vagal tone, and respiration: Within- and between-individual relations. Psychophysiology, 30, 486-495.

Hayano, J., Sakakibara, Y., Yamada, A., Mukai, S., Fujinami, T., Yokoyama, K., et al. (1991). Accuracy of assessment of cardiac vagal tone by heart rate variability in normal subjects. American Journal of Cardiology, 67, 199-204.

Houtveen, J. H., Rietveld, S., \& De Geus, E. J. C. (2002). Contribution of tonic vagal modulation of heart rate, central respiratory drive, respiratory depth, and respiratory frequency to respiratory sinus arrhythmia during mental stress and physical exercise. Psychophysiology, 39, 427-436.

Huikuri, H. V., Pikkujamsa, S. M., Airaksinen, K. E. J., Ikaheimo, M. J., Rantala, A. O., Kauma, H., et al. (1996). Sex-related differences in autonomic modulation of heart rate in middle-aged subjects. Circulation, 94, 122-125.

Jaccard, J., Turrisi, R., \& Wan, C. K. (1990). Interaction effects in multiple regression. In M. S. Lewis Beck (Series Ed.), Series: Quantitative applications in the social sciences (Vol. 72). Newbury Park, CA: Sage.

Korkushko, O. V., Shatilo, V. B., Plachinda, Y. I., \& Shatilo, T. V. (1991). Autonomic control of cardiac chronotropic function in man as a function of age: Assessment by power spectral analysis of heart rate variability. Journal of the Autonomic Nervous System, 32, 191-198.

Kristal-Boneh, E., Raifel, M., Froom, P., \& Ribak, J. (1995). Heart rate variability in health and disease. Scandinavian Journal of Work and Environmental Health, 21, 85-95.

Liao, D., Barnes, R. W., Chambless, L. E., Simpson, R. J., Sorlie, P., \& Heiss, G. (1995). Age, race, and sex differences in autonomic cardiac function measured by spectral analysis of heart rate variability-The ARIC Study. American Journal of Cardiology, 76, 906-912.

Liao, D., Cai, J., Rosamond, W. D., Barnes, R. W., Hutchinson, R. G., Whitsel, E. A., et al. (1997). Cardiac autonomic function and incident coronary heart disease: A population-based case-cohort study: The ARIC Study. American Journal of Epidemiology, 145, 696-706.

Mäkimattila, S., Schlenzka, A., Mäntysaari, M., Bergholm, R., Summanen, P., Saar, P., et al. (2000). Predictors of abnormal autonomic function measured by frequency domain analysis of heart rate variability and conventional tests in patients with Type 1 diabetes. Diabetes Care, 23, $1686-1693$.

Manuck, S. B., Kasprowicz, A. L., \& Muldoon, M. F. (1990). Behaviorally-evoked cardiovascular reactivity and hypertension: Conceptual issues and potential associations. Annals of Behavioral Medicine, 12, 17-29.

Marshall, P. J., \& Stevenson-Hinde, J. (1998). Behavioral inhibition, heart period, and respiratory sinus arrhythmia in young children. Developmental Psychobiology, 33, 283-292.

Massin, M., \& von Bernuth, G. (1997). Normal ranges of heart rate variability during infancy and childhood. Pediatric Cardiology, 18, 297-302.

Matthews, K. A., Salomon, K., Kenyon, K., \& Allen, M. T. (2002). Stability of children's and adolescents' hemodynamic responses to psychological challenge: A three-year longitudinal study of a multiethnic cohort of boys and girls. Psychophysiology, 39, 826-834.

Murphy, J. K., Alpert, B. S., Walker, S. S., \& Willey, E. S. (1991). Children's cardiovascular reactivity: Stability of racial differences and relation to subsequent blood pressure over a one-year period. Psychophysiology, 28, 447-457.

Newman, J. D., McGarvey, S. T., \& Steele, M. S. (1999). Longitudinal association of cardiovascular reactivity and blood pressure in Samoan adolescents. Psychosomatic Medicine, 61, 243-249.

Parker, F. C., Croft, J. B., Cresanta, J. L., Freedman, D. S., Burke, G. I., Webber, L. S., et al. (1987). The association between cardiovascular 
response tasks and future blood pressure levels in children: Bogalusa Heart Study. American Heart Journal, 113, 1174-1179.

Peterson, A. C., Crockett, L., Richards, M., \& Boxer, A. (1988). A self-report measure of pubertal status: Reliability, validity, and initial norms. Journal of Youth and Adolescence, 17, 117-133.

Porges, S. W. (1991). Vagal tone: An autonomic mediator of affect. In J. Garber \& K. A. Dodge (Eds.), The development of emotion regulation and dysregulation (pp. 111-128). Cambridge, England: Cambridge University Press.

Porges, S. W. (1992). Autonomic regulation and attention. In B. A. Campbell, H. Hayne, \& R. Richardson (Eds.), Attention and information processing in infants and adults. (pp. 30-60). Hillsdale, NJ: Erlbaum.

Porges, S. W. (1995). Orienting in a defensive world: Mammalian modifications of our evolutionary heritage: A polyvagal theory. Psychophysiology, 32, 301-318.

Porges, S. W. (2001). The polyvagal theory: Phylogenetic substrates of a social nervous system. International Journal of Psychophysiology, 42, $1314-1326$.

Porges, S. W., Doussard-Roosevelt, J. A., Portales, A. L., \& Greenspan, S. I. (1996). Infant regulation of the vagal "brake" predicts child behavior problems: A psychobiological model of social behavior. Developmental Psychobiology, 29, 697-712.

Porges, S. W., Doussard-Roosevelt, J. A., Portales, A. L., \& Suess, P. E. (1994). Cardiac vagal tone: Stability and relation to difficultness in infants and three-year-olds. Developmental Psychobiology, 27, 289300.

Prentice, D. A., \& Miller, D. T. (1992). When small effects are impressive. Psychological Bulletin, 112, 160-164.

Rossy, L. A., \& Thayer, J. F. (1998). Fitness and gender-related differences in heart period variability. Psychosomatic Medicine, 60, 773-781.

Sahar, T., Shalev, A. Y., \& Porges, S. W. (2001). Vagal modulation of responses to mental challenge in posttraumatic stress disorder. Biological Psychiatry, 49, 637-643.

Salomon, K., Matthews, K. A., \& Allen, M. T. (2000). Patterns of sympathetic and parasympathetic reactivity in a sample of children and adolescents. Psychophysiology, 37, 842-849.

Sevre, K., Lefrandt, J. D., Nordby, G., Os, I., Mulder, M., Gans, R. O. B., et al. (2001). Autonomic function in hypertensive and normotensive subjects: The importance of gender. Hypertension, 37, 1351-1356.

Sherwood, A., Allen, M. T., Fahrenberg, J., Kelsey, R. M., Lovallo, W. R., \& van Doornen, L. J. P. (1990). Methodological guidelines for impedance cardiography. Psychophysiology, 27, 1-23.

Singh, J. P., Larson, M. G., Tsuji, H., Evans, J. C., O’Donnell, C. J., \& Levy, D. (1998). Reduced heart rate variability and new-onset hypertension: Insights into pathogenesis of hypertension: The Framingham Heart Study. Hypertension, 32, 293-297.

Sinnreich, R., Kark, J. D., Friedlander, Y., Sapoznikov, D., \& Luria, M. H. (1998). Five minute recordings of heart rate variability for population studies: Repeatability and age-sex characteristics. Heart, 80, 156-162.

Sloan, R. P., Shapiro, P. A., Bagiella, E., Fishkin, P. E., Gorman, J. M., \& Myers, M. M. (1995). Consistency of heart rate and sympathovagal reactivity across different autonomic contexts. Psychophysiology, 32, $452-459$

Sloan, R. P., Shapiro, P. A., Bagiella, E., Gorman, J. M., \& Bigger, J. T. (1995). Temporal stability of heart period variability during a resting baseline and in response to psychological challenge. Psychophysiology, 32, 191-196.

Sloan, R. P., Shapiro, P. A., Bigger, J. T., Bagiella, E., Steinman, R. C., \& Gorman, J. M. (1994). Cardiac autonomic control and hostility in healthy subjects. American Journal of Cardiology, 74, 298-300.

Stein, P. K., Kleiger, R. E., \& Rottman, J. N. (1997). Differing effects of age on heart rate variability in men and women. American Journal of Cardiology, 80, 302-305.

Tabachnick, B. G., \& Fidell, L. S. (1989). Using multivariate statistics. New York: Harper \& Row.

The Task Force of the European Society of Cardiology and the North American Society of Pacing and Electrophysiology. (1996). Heart rate variability: Standards of measurement, physiological interpretation, and clinical use. Circulation, 93, 1043-1065.

Taylor, J. A., Myers, C. W., Halliwill, J. R., Seidel, H., \& Eckberg, D. L. (2001). Sympathetic restraint of respiratory sinus arrhythmia: Implications for vagal-cardiac tone assessments in humans. American Journal of Physiology-Heart and Circulatory Physiology, 280, H2804-H2814.

Thayer, J. F., Friedman, B. H., \& Borkovec, T. D. (1996). Autonomic characteristics of generalized anxiety disorder and worry. Biological Psychiatry, 39, 255-266.

Thayer, J. F., Friedman, B. H., Borkovec, T. D., Johnson, B. J., \& Molina, S. (2000). Phasic heart period reactions to cued threat and nonthreat stimuli in generalized anxiety disorder. Psychophysiology, 37, 361-368.

Treiber, F. A., Musante, L., Kapuku, G., Davis, C., Litaker, M., \& Davis, H. (2001). Cardiovascular (CV) responsivity and recovery to acute stress and future $\mathrm{CV}$ functioning in youth with family histories of CV disease: A 4-year longitudinal study. International Journal of Psychophysiology, $41,65-74$.

Tsuji, H., Larson, M. G., Venditti, F. J., Manders, E. S., Evans, J. C., Feldman, C. L., et al. (1996). Electrophysiology/pacing: Impact of reduced heart rate variability on risk for cardiac events: The Framingham Heart Study. Circulation, 94, 2850-2855.

Umetani, K., Singer, D. H., McCraty, R., \& Atkinson, M. (1998). Twentyfour hour time domain heart rate variability and heart rate: Relations to age and gender over nine decades. Journal of the American College of Cardiology, 31, 593-601.

van Ravenswaaij-Arts, C. M., Kollee, L. A., Hopman, J. C., Stoelinga, G. B., \& van Geijn, H. P. (1993). Heart rate variability. Annals of Internal Medicine, 118, 436-447.

Yeh, S., Forsythe, A., \& Hon, E. H. (1973). Quantification of fetal heart beat-to-beat differences. Obstetrics \& Gynecology, 41, 355-363.

Yeragani, V. K., Pohl, R., Berger, R., Balon, R., \& Srinivasan, K. (1994). Relationship between age and heart rate variability in supine and standing postures: A study of spectral analysis of heart rate. Pediatric Cardiology, 15, 14-20. 